\title{
Standard of usual care defines effectiveness of early goal directed therapy
}

\author{
Vikram Mukherjee ${ }^{1}$, Jan Bakker ${ }^{1,2,3,4}$ \\ ${ }^{1}$ Department of Pulmonology and Critical Care, NYU Langone Health, NYU School of Medicine, New York, NY, USA; ${ }^{2}$ Department of Intensive \\ Care Adults, Erasmus MC University Medical Center, Rotterdam, The Netherlands; ${ }^{3}$ Department of Pulmonology and Critical Care, Columbia \\ University Medical Center, New York, NY, USA; ${ }^{4}$ Department of Intensive Care, Pontificia Universidad Catolica de Chile, Santiago, Chile \\ Correspondence to: Jan Bakker, MD, PhD, FCCM, FCCP. Bellevue Hospital, 462 First Avenue NBV-10W15. New York, NY 10016, USA. \\ Email: jb3387@cumc.columbia.edu; Jan.Bakker@nyumc.org. \\ Provenance: This is an invited article commissioned by the Section Editor Dr. Guo-Wei Tu (Department of Critical Care Medicine, Zhongshan \\ Hospital, Fudan University, Shanghai, China). \\ Comment on: Ding XF, Yang ZY, Xu ZT, et al. Early goal-directed and lactate-guided therapy in adult patients with severe sepsis and septic shock: a \\ meta-analysis of randomized controlled trials. J Transl Med 2018;16:331.
}

Submitted Aug 29, 2019. Accepted for publication Sep 12, 2019.

doi: $10.21037 / \mathrm{atm} .2019 .09 .71$

View this article at: http://dx.doi.org/10.21037/atm.2019.09.71

Sepsis is a leading cause of mortality and morbidity globally (1). Unfortunately, given wide variations in practices and resources available, there are significant variations in outcomes among different regions of the world (2). Ever since the seminal study was done by Rivers et al. demonstrated that patients with severe sepsis and septic shock warrant early, goal-directed care (3), there has been a paradigm shift on how this disease state is managed.

Ding et al. provide an interesting perspective into existing data surrounding the efficacy of early goal directed therapy (EGDT) (4). In their meta-analysis, the authors describe 16 randomized control trials (RCTs) comparing mortality between EGDT and usual care (UC). Of note, the selected studies offer a thought-provoking insight into the wide variations in UC outcomes of severe sepsis and septic shock globally. As demonstrated in the accompanying picture, traditionally well-resourced countries demonstrate impressive outcomes in their UC arms (Figure 1). On the contrary, mortality in the UC arms in other parts of the world has been shown to significantly higher.

It is in this context that we should compare the effectiveness of EGDT. In the three large RCTs comparing EGDT to UC (ARISE, PROCESS and PROMISE) (5-7), the mortality rates in the UC arms were between $18-30 \%$. It is also in these studies where there was no marked difference between EGDT and UC. While this can be misinterpreted as "EGDT is not effective", the more accurate way of explaining these findings is that these centers were implementing early, targeted care in their UC arms, thus negating any benefit that EGDT might show. As demonstrated by Nguyen et al. (8), the UC arms in these three RCTs all received fluids (3.9-4.3L in the first $6 \mathrm{~h}$ ), hemodynamic support ( $44.1 \%$ to $57.8 \%$ in the first $6 \mathrm{~h}$ ) and had antibiotic administration as a prerequisite to study enrolment. Additionally, the baseline $\mathrm{ScvO}_{2}$ levels in these three studies are similar to the post-resuscitation $\mathrm{ScvO}_{2}$ in the EGDT arm of the Rivers trial- further evidence that patients were well resuscitated in both UC and EGDT arms of these trials. It is probably because of these measures that there was no significant difference in outcomes when comparing EGDT to UC in these trials.

When we take this in contrast to studies comparing EGDT to UC/LGT in less resourced areas of the world, the benefits of EGDT are marked. Absolute risk reduction in death ranges from $18-20 \%(9,10)$ when EGDT is applied to such less resourced areas. In these studies, UC was markedly different compared to EGDT-amount of fluid administered and delays in vasopressor administration; this might have led to an improvement in outcomes when protocolized care was initiated.

This reflection is also emphasized by the forest plot in the Figure 4, where EGDT shows superiority to UC prior 


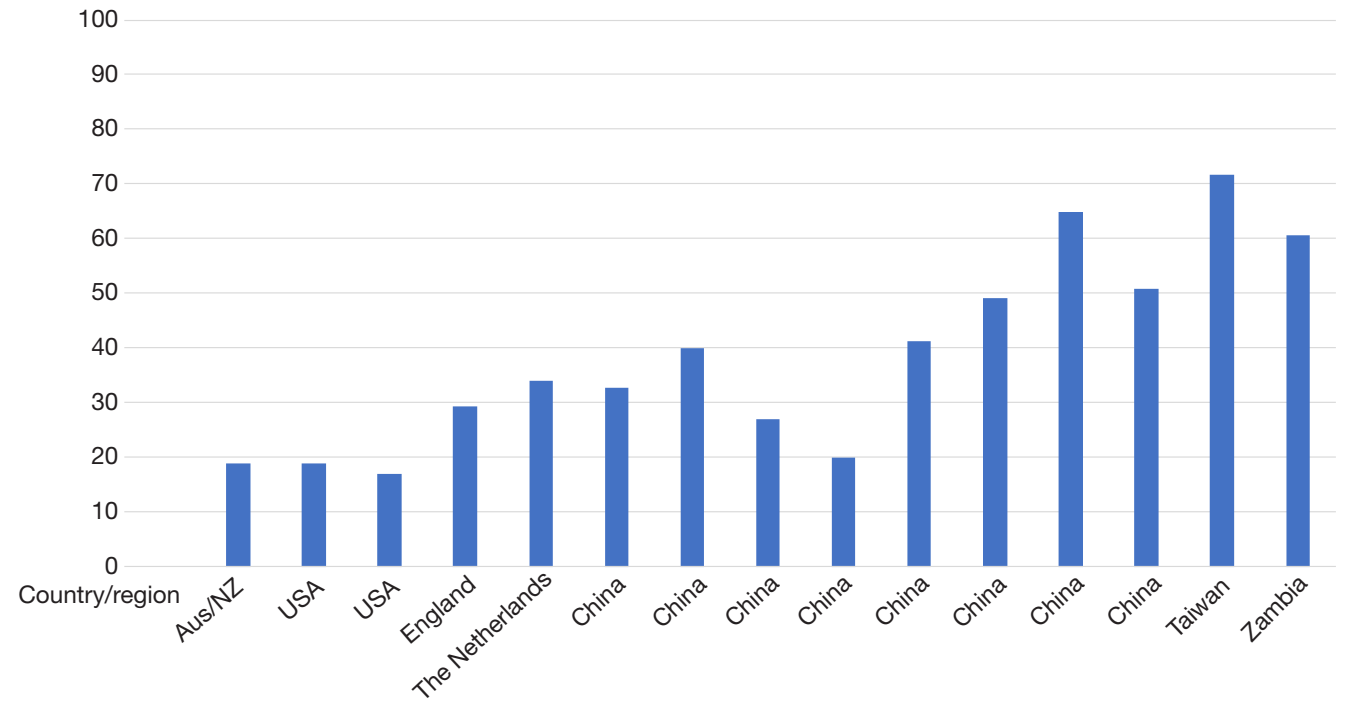

Figure 1 Variations in mortality rates in the usual care arms of randomized controlled trials comparing usual care to early goal directed therapy in patients with severe sepsis or septic shock [the results of the study by Rivers et al. (3) has been left out of this figure as this is seen as the landmark study].

to the SSC 2012 guidelines. The SSC 2012 guidelines were a resource document providing recommendations on early management of sepsis and septic shock; there is ample evidence that implementation of these guidelines improves outcomes $(11,12)$. The absence of superiority of EGDT over UC is most likely because of an improvement in the latter due to implementation of the SSC guidelines.

The Ding et al. meta-analysis therefore summarizes an obvious fact- protocolized care will improve outcomes if UC is suboptimal. As Emergency room and ICUs across the world ramp up their efforts to be able to identify and manage unstable patients rapidly-irrespective of their underlying etiology, be it trauma, sepsis or acute coronary syndromes- we hope to see the differences between protocolized care and UC continue to narrow.

\section{Acknowledgments}

None.

\section{Footnote}

Conflicts of Interest: The authors have no conflicts of interest to declare.

Ethical Statement: The authors are accountable for all aspects of the work in ensuring that questions related to the accuracy or integrity of any part of the work are appropriately investigated and resolved.

\section{References}

1. Adhikari NK, Fowler RA, Bhagwanjee S, et al. Critical care and the global burden of critical illness in adults. Lancet 2010;376:1339-46.

2. Sakr Y, Jaschinski U, Wittebole X, et al. Sepsis in Intensive Care Unit Patients: Worldwide Data From the Intensive Care over Nations Audit. Open Forum Infect Dis 2018;5:ofy313.

3. Rivers E, Nguyen B, Havstad S, et al. Early goal-directed therapy in the treatment of severe sepsis and septic shock. N Engl J Med 2001;345:1368-77.

4. Ding XF, Yang ZY, Xu ZT, et al. Early goal-directed and lactate-guided therapy in adult patients with severe sepsis and septic shock: a meta-analysis of randomized controlled trials. J Transl Med 2018;16:331.

5. ARISE Investigators, ANZICS Clinical Trials Group, Peake SL, et al. Goal-directed resuscitation for patients with early septic shock. N Engl J Med 2014;371:1496-506.

6. Mouncey PR, Osborn TM, Power GS, et al. Trial of early, goal-directed resuscitation for septic shock. N Engl J Med 2015;372:1301-11.

7. ProCESS Investigators, Yealy DM, Kellum JA, et al. A randomized trial of protocol-based care for early septic 
shock. N Engl J Med 2014;370:1683-93.

8. Nguyen HB, Jaehne AK, Jayaprakash N, et al. Early goaldirected therapy in severe sepsis and septic shock: insights and comparisons to ProCESS, ProMISe, and ARISE. Crit Care 2016;20:160.

9. Lin SM, Huang CD, Lin HC, et al. A modified goaldirected protocol improves clinical outcomes in intensive care unit patients with septic shock: a randomized controlled trial. Shock 2006;26:551-7.

10. Chen ZQ, Jin YH, Chen H, et al. [Early goal-directed

Cite this article as: Mukherjee V, Bakker J. Standard of usual care defines effectiveness of early goal directed therapy. Ann Transl Med 2019;7(Suppl 8):S352. doi: 10.21037/ atm.2019.09.71 therapy lowers the incidence, severity and mortality of multiple organ dysfunction syndrome]. Nan Fang Yi Ke Da Xue Xue Bao 2007;27:1892-5.

11. Ferrer R, Artigas A, Levy MM, et al. Improvement in process of care and outcome after a multicenter severe sepsis educational program in Spain. JAMA 2008;299:2294-303.

12. Li ZQ, Xi XM, Luo X, et al. Implementing surviving sepsis campaign bundles in China: a prospective cohort study. Chin Med J (Engl) 2013;126:1819-25. 